\title{
A Comparison of Intradialytic versus Out-of-Clinic Exercise Training Programs for Hemodialysis Patients
}

\author{
Hsin-Yu Fang ${ }^{a}$ Brett T. Burrows ${ }^{a}$ Alexis C. King ${ }^{a}$ Kenneth R. Wilund ${ }^{a, b}$ \\ a Department of Kinesiology and Community Health, University of Illinois at Urbana-Champaign, Urbana, IL, USA; \\ ${ }^{b}$ Division of Nutritional Sciences, University of Illinois at Urbana-Champaign, Urbana, IL, USA
}

\section{Keywords}

End-stage kidney disease $\cdot$ Hemodialysis $\cdot$ Exercise $\cdot$ Physical activity

\begin{abstract}
Background: Physical inactivity is prevalent and linked with a variety of unfavorable clinical outcomes in hemodialysis patients. To increase physical activity (PA) and improve quality of life in this population, intradialytic and out-of-clinic exercise interventions have been implemented in many studies. However, there is still a lack of consensus in the literature on which type of exercise intervention is more feasible and effective. Summary: This review provides a brief overview of intradialytic and out-of-clinic exercise protocols utilized in previous studies. We also examine data related to the feasibility of each approach, and their efficacy for improving cardiovascular health, muscle mass, strength, and physical function. Key Messages: The benefits from most intradialytic and out-of-center exercise training interventions published to date have been modest or inconsistent. Furthermore, neither appears to provide a significant advantage over the other in terms of benefits for cardiovascular health, muscle mass, strength, and physical function. A significant concern is that most intradialytic and out-of-center exercise interventions are mandated exercise prescriptions that include either endurance or resistance training exercises, per-
\end{abstract}

formed at low-moderate intensities, for a total of $60-135 \mathrm{~min}$ of exercise/week. This volume, intensity, and variety of exercise are far less than what is recommended in most PA guidelines. This type of structured activity is also boring for most patients. To enhance the effectiveness of exercise interventions, we suggest using the intradialytic period to provide patients guidance on how they can best incorporate more activity into their lives, based on their individual needs and barriers.

(c) 2019 S. Karger AG, Basel

\section{Introduction}

Patients with renal failure undergoing maintenance hemodialysis (HD) therapy typically have very low levels of physical activity (PA), and this is associated with greatly increased morbidity and mortality [1]. To increase activity levels, clinicians and researchers have experimented with different types of interventions, including both incenter ("intradialytic") and out-of-clinic ("interdialytic") exercise programs. While any type of PA is likely to have benefits, a common perception is that intradialytic exercise is more feasible to implement, while out-of-clinic programs provide patients more options from which to choose. However, there is no consensus in the literature regarding which type provides the greatest overall bene-

\section{KARGER}

(C) 2019 S. Karger AG, Basel

E-Mail karger@karger.com

www.karger.com/bpu
Kenneth R. Wilund, PhD

Department of Kinesiology and Community Health

University of Illinois at Urbana-Champaign

906 S. Goodwin Avenue, Urbana, IL 61801 (USA)

E-Mail kwilund@illinois.edu 
fits, and this has left clinicians unsure about how to best prescribe exercise for their patients. The primary aim of this review is to examine data in the published literature to compare the feasibility and effectiveness of intradialytic versus out-of-clinic exercise and PA programs. Because there are clear advantages and disadvantages to each approach, we propose a more personalized strategy for exercise prescription that provides patients more autonomy to determine when, where, and what types of activities in which they choose to engage.

\section{Comparison of Intradialytic and Out-of-Clinic Exercise Protocols}

\section{Intradialytic Exercise Protocols}

Both endurance and resistance exercise training protocols have been widely utilized during dialysis. Intradialytic cycling is the primary mode of endurance exercise implemented in most published research studies. This involves patients pedaling on a cycle ergometer during dialysis, with the intensity generally controlled by adjusting the resistance to a flywheel or revolutions per minute. This is typically performed 2-3 times per week at a lowmoderate intensity, with average training durations around $30 \mathrm{~min} /$ session [2].

Intradialytic resistance training has also been used as the primary mode of exercise in many studies [3]. This usually includes several leg exercises performed with ankle weights or resistance bands, 1-3 sets per exercise, 2-3 times per week, at a low to moderate intensity/resistance. If upper body activities are included, the nonaccess arm may be exercised during dialysis, while the access arm may be exercised before or after treatment. A few resistance training studies have utilized weight training machines placed at the end of the patients bed or chair $[4,5]$, while some have used machines placed in the waiting room of the dialysis clinic $[6,7]$, so would most appropriately be termed "peridialytic" training.

\section{Other Forms of Intradialytic Physical Activity}

Other activity-related protocols that have been incorporated into the intradialytic period include neuromuscular electrical stimulation [8], respiratory muscle training [9], Zumba [10], and laughter yoga [11]. Protocols such as these are generally very low intensity, or utilize small muscle groups, so may be best categorized as PA instead of exercise. Nonetheless, the diversity of intradialytic training programs in the published literature indicates there are more options for intradialytic PA than commonly perceived. Yet, implementing these types of activities in the clinic are challenging, as will be discussed below.

\section{Out-of-Clinic Exercise Protocols}

In theory, there is no limit to the types of activities that can be performed in out-of-center exercise programs. Despite this, few published studies use out-of-clinic exercise as the primary mode of training [12]. Most published out-of-clinic exercise protocols fall into 3 categories: (1) home-based walking programs; (2) resistance training in a fitness center; and (3) combined programs that include aerobic, resistance, and/or educational components. Home-based walking programs typically include individualized work rates based on baseline functional capacities of patients, with a total duration of 15-45 min/day, starting at a low intensity, progressing in time and/or intensity during the intervention $[13,14]$. Of course, HD patients also engage in unprescribed exercise or lifestyle PA outside of the clinic (e.g., walking), but the volume and intensity of these activities are generally well below recommended levels and may vary significantly based on patient comorbidities, geographic location, and other factors $[1,15]$.

Out-of-clinic resistance training has generally been supervised and may include 5-10 exercises targeting lower-limb or whole-body exercises using light weights, resistance bands, or weight machines. The training volume and intensity may progress from 1 to 5 sets/exercise, and from a low to moderate load, depending on the ability of the patient [16-18]. Upper body exercises may be prescribed at a lower intensity to reduce concerns with patients lifting heavy loads with their vascular access arm, despite limited evidence of risks in doing so [19].

\section{Safety and Feasibility}

\section{Intradialytic Exercise}

Most published exercise interventions in HD patients have utilized intradialytic exercise because it is considered the most feasible to implement. During dialysis, patients are considered a somewhat "captive audience," given they have little else to do during their treatment. The clinic staff is also available to help implement the program. This should lead to higher exercise compliance and less patient burden. However, there is little empirical evidence to support this, as exercise programs are found in $<10 \%$ of HD clinics worldwide, and enrollment and adherence in these are generally low $[20,21]$. There are also 
Table 1. Hypothetical advantages and concerns with intradialytic and out-of-center exercise

\begin{tabular}{|c|c|c|c|}
\hline \multicolumn{2}{|l|}{ Intradialytic exercise } & \multicolumn{2}{|l|}{ Out-of-center exercise } \\
\hline proposed advantages & proposed disadvantages & proposed advantages & proposed disadvantages \\
\hline Time efficient & $\begin{array}{l}\text { Limited choice of exercise } \\
\text { types }\end{array}$ & $\begin{array}{l}\text { Unlimited choice of } \\
\text { exercise types }\end{array}$ & $\begin{array}{l}\text { Limited access to exercise } \\
\text { facilities }\end{array}$ \\
\hline $\begin{array}{l}\text { Higher compliance } \\
\text { (easy to monitor) }\end{array}$ & Limited exercise intensity & $\begin{array}{l}\text { Potentially higher } \\
\text { exercise intensity }\end{array}$ & $\begin{array}{l}\text { Lower or unreliable } \\
\text { compliance }\end{array}$ \\
\hline Less patient burden & Limited mobility & Unlimited mobility & $\begin{array}{l}\text { Higher cost for trained } \\
\text { personnel }\end{array}$ \\
\hline \multirow[t]{2}{*}{$\begin{array}{l}\text { Safety } \\
\text { (exercise monitoring) }\end{array}$} & $\begin{array}{l}\text { Potential hemodynamic } \\
\text { instability }\end{array}$ & $\begin{array}{l}\text { Less burden on } \\
\text { clinic staff }\end{array}$ & $\begin{array}{l}\text { Less safe } \\
\text { (unsupervised exercise) }\end{array}$ \\
\hline & $\begin{array}{l}\text { Potential exacerbation of } \\
\text { muscle catabolism }\end{array}$ & & $\begin{array}{l}\text { Low perceived self- } \\
\text { efficacy of patients }\end{array}$ \\
\hline
\end{tabular}

many inherent disadvantages associated with exercising while confined to a chair or bed. In particular, the intensity and variety of activities that can be performed during dialysis are limited, with the most practical being cycling and lower-body resistance training. There are also concerns that intradialytic exercise may exacerbate hemodynamic instability and other issues that are common during treatment, including intradialytic hypertension, hypotension, cramping, and fatigue, though there is little evidence for this [19]. Moreover, since dialysis is a catabolic process, there is a theoretical risk that the increased energy expenditure associated with intradialytic exercise could exacerbate muscle protein breakdown [22], though this has not been directly tested.

\section{Out-of-Clinic Exercise}

Out-of-clinic exercise programs have the potential to incorporate a greater variety of activities. This was highlighted in a study by Greenwood et al. [23] in which patients attended biweekly supervised exercise and education sessions and performed once-weekly home-based exercise that included endurance, resistance, and balance and flexibility training. Out-of-clinic exercise has the potential to utilize greater volumes and higher intensity exercise, if the activity is supervised. However, many out-ofclinic programs are unsupervised, as this makes them more feasible by reducing the burden on the clinic staff. Unsupervised programs have several significant disadvantages related to patient safety, compliance, adherence, and low-self-efficacy [24] (Table 1). Perhaps because of these concerns, unsupervised out-of-clinic exercise programs typically prescribe a low volume and intensity of exercise. Examples of this include: (1) the EXCITE trial [14] in which HD patients were prescribed $\sim 60 \mathrm{~min} /$ week of low-intensity walking and (2) the Life Readiness Program [25] in which patients were instructed to accumulate $30 \mathrm{~min}$ of light-intensity lifestyle physical activities (e.g., yardwork, shopping, house cleaning, etc.).

In summary, there is no consensus in the literature as to whether intradialytic or out-of-clinic exercise is safer or more feasible to implement. There are advantages and concerns with each, depending in part on the types of activity that are prescribed (Table 1). Below, we will describe the relative efficacy of out-of-clinic and intradialytic exercise on cardiovascular and muscle/physical function-related outcomes.

\section{Effects on Cardiovascular Health and Physical Function}

\section{Intradialytic Endurance Exercise}

The overwhelming majority of endurance training interventions in HD have utilized intradialytic cycling [26], and the primary outcomes targeted have included measures of cardiovascular health, physical function, and aerobic capacity. In a recent meta-analysis of 24 randomized controlled trials (RCTs) of intradialytic endurance exercise, both systolic and diastolic blood pressure were significantly reduced, but the magnitude of the changes was small $(\Delta$ systolic blood pressure $=-0.27 \mathrm{~mm} \mathrm{Hg}(-0.50$ to $0.04 ; p=0.02) ; \Delta$ diastolic blood pressure $=-0.24(-0.47$ to $-0.01 ; p=0.04)$, so the clinical relevance of these data is unclear. Furthermore, a meta-analysis by Young et al. 
[2] reported that intradialytic cycling had no significant effect on BP and arterial stiffness [27]. The effects of intradialytic endurance exercise on physical function and aerobic capacity are not only generally positive but also inconsistent. For example, several meta-analyses and systematic reviews indicate statistically significant improvements in physical function and aerobic capacity (e.g., 6-min walk test, gait speed, $\mathrm{VO}_{2}$ peak, etc.) after 3-6 months of intradialytic endurance exercise $[2,3,12,26]$. However, the magnitude of the changes in physical function is generally modest. Furthermore, 1 meta-analysis indicated that aerobic capacity does not improve with intradialytic cycling [2], while another indicates aerobic capacity only improves following interventions of $>6$ months [26], which are rare.

\section{Out-of-Clinic Endurance Exercise}

While only a few studies have evaluated the efficacy of out-of-clinic endurance training, this includes 2 of the largest exercise-related RCTs in HD patients conducted to date $[13,14]$. Both of these trials included a 6-month, low-moderate intensity home-based walking intervention. While the walking intervention in the study by Manfredini et al. [14] produced modest improvements in performance on the 6-min walk test, the intervention from Koh et al. [13] did not. Unfortunately, neither intervention improved measures of cardiovascular health, including BP $[13,14]$, nor arterial stiffness [13].

Taken together, data from intradialytic and out-ofcenter endurance training studies suggest: (1) both modestly improve measures of physical function, though the data are not entirely consistent [13]; (2) neither significantly improves markers of cardiovascular health, including BP, arterial stiffness, and perhaps aerobic capacity $\left(\mathrm{VO}_{2}\right.$ peak). In other words, despite hypothetical advantages and concerns for both, neither has a distinct advantage in terms of producing consistent, robust benefits across multiple health-related outcomes. There may be several reasons for this, including the low volume and intensity of exercise that is generally used in both intradialytic and out-of-clinic endurance training interventions $[2,28]$. The failure to improve measures of cardiovascular health may be a consequence of cardiovascular complications of renal failure, such as chronic volume overload, calcification, and endothelial dysfunction. These comorbidities may cause vascular remodeling that limits the vessels ability to respond to the intervention, regardless of whether the exercise is performed during dialysis or outside of the clinic.

\section{Effects on Muscle Mass, Strength, and Function}

\section{Intradialytic Resistance Training}

The most effective method for improving muscle mass, strength, and function is with whole body resistance training in which muscles are progressively overloaded using increasing weights or resistance over time. Unfortunately, this is difficult to do in patients confined to a dialysis chair or bed. As mentioned previously, the majority of intradialytic resistance training protocols have included low- to moderate-intensity exercises using ankle weights or elastic bands. The most consistent benefit in these studies has been improvements in muscle strength, while changes in muscle mass and physical function have been modest or inconsistent [29-33].

To increase intradialytic training loads, a few studies have used specially designed weight training machines that fit at the end of a patient's dialysis chair or bed [4, 5], while a few others have utilized weight machines to do "peridialytic" training in the waiting room before or after dialysis [6, 7] (Table 1). While these machines may increase the load during any particular exercise, most clinics do not have space for bulky weight training equipment, so this reduces the types of exercises that can be performed on machines. For example, in studies by Kirkman et al. [4] and Dong et al. [6], the resistance training protocols consisted of only one type of exercise (leg press). Kirkman et al. [4] reported both muscle volume and strength improved after 12 weeks of training, but physical function (6-min walk test) did not improve. By contrast, Dong et al. [6] showed 6 months of a similar training protocol had no effects on lean mass and muscle strength. Kopple et al. [7] also conducted a 21-week resistance training intervention that incorporated 4 different types of leg exercises on weight training machines in the clinic lobby. Despite this increased volume of exercise, there were no changes in body composition (strength and physical function were not assessed). Taken together, these data indicate few differences in benefits from intradialytic resistance training when using light weights, resistance bands, or weight training machines.

\section{Out-of-Center Resistance Training}

In theory, out-of-center resistance training should provide an opportunity for a greater volume and intensity of resistance exercise to be performed, and there are several good examples of this $[16,18]$. In one of the most robust resistance training interventions to 


Endurance training
Intradialytic cycling or home-based walking
program $60-135$ min/week
low to moderate intensity training

Fig. 1. Comparison of traditional intradialytic and out-in-clinic exercise programs versus PA recommendations. Standard intradialytic and out-of-clinic exercise programs typically include a single type of mandated exercise (aerobic or resistance training), conducted 2-3 days per week, for 30-45 min, at a low - moderate intensity. By contrast, the PA recommendations for adults with chronic health conditions and disabilities [34] call for a greater volume and intensity of exercise and also encourage a much greater variety of activities. This includes aerobic activity most days of the week and whole-body resistance training at least 2 days per week. Other activities including balance, flexibility, and multicomponent activities (e.g., dancing and sports) are also recommended as abilities allow. If unable to meet these guidelines, all

date in HD patients, Headley et al. [18] evaluated the efficacy of a 12-week progressive, whole-body resistance training protocol using weight machines in a fitness center. While muscle strength and physical function improved, changes in muscle mass were unfortunately not assessed. Molsted et al. [16] also conducted a 16-week, high-intensity, progressive resistance training protocol consisting of 3 different leg exercises performed on machines at a fitness center. While both muscle strength and objective physical function measures improved, once again, muscle mass was not. Taken together, these data suggest that HD patients may have an anabolic resistance to the effects of exercise training, though additional studies using progressive resistance training protocols are needed to confirm this. people are encouraged to move more and sit less. We contend that the standard approaches that have been used to prescribe exercise in dialysis patients should be reconsidered. Instead of providing mundane, one-size-fits-all, mandated exercise prescriptions to HD patients, the intradialytic period should be used to provide guidance on how to incorporate PA into their lives by any means possible. This should include intradialytic exercise, low-intensity "lifestyle" activities, progression to higher-intensity activities outside of the clinic, and even recreational activities for patients that are willing and able to participate. Indeed, this revised approach is congruent with evidence-based PA guidelines for patients with chronic diseases and disabilities [34] designed to optimize these individuals' health and quality of life. PA, physical activity.

\section{Discussion}

It is widely recognized that the health and quality of life of HD patients would improve by increasing their PA levels, but there is little consensus on how best to accomplish this. An active debate in the literature is whether intradialytic or out-of-center exercise is more feasible or efficacious. While there are inherent advantages and concerns with both approaches (Table 1), exercise programs in HD clinics of any kind are exceedingly rare $[20,21]$, participation rates in the existing programs are low, and physical inactivity remains a hallmark of the disease [1]. Moreover, even in highly supervised RCTs, regardless of the frequency, intensity, type, or timing of the exercise (intradialytic or out-ofcenter), the overall benefits demonstrated in most studies have been modest. 
There are several significant concerns with almost all of the intradialytic and out-of-clinic exercise interventions conducted to date. Primarily, the volume and intensity of exercise performed has been well below what is recommended by standard PA guidelines, including those for older adults and individuals with chronic diseases [34] (Fig. 1). To improve one's health through PA, these guidelines recommend 150-300 min/week of moderate intensity endurance exercise, plus 2 or more days of resistance training. Additional activities throughout the week such as balance and flexibility training are also recommended. By contrast, most exercise interventions in HD patients, regardless of whether it is prescribed during dialysis or outside of the clinic, have included a single mode of exercise (either endurance or resistance training), at a light moderate intensity, on 2-3 days/week, usually totaling between 60 and $135 \mathrm{~min} /$ week. Despite this low volume and intensity, patient participation and adherence to the prescribed activities are poor, and dropout rates are high [35].

This strongly suggests that new approaches to implementing exercise in HD patients should be considered. The mundane, one-size-fits-all approaches that we typically prescribe (e.g., intradialytic cycling or at-home walking programs) have failed to address the wants or needs of a diverse patient population. Instead, individualized interventions should be designed to allow patients more autonomy to select types of activities in which they choose to engage. This will include providing patients guidance on how to incorporate PA into their lives by any means possible, including low-intensity "lifestyle" activities, intradialytic exercises, and progression to higher-intensity activities outside of the clinic, and even recreational activities for patients that are willing and able to participate. Factors that influence a patient's ability to exercise, including comorbidities [24], geographic location [15], and other barriers, need to be considered when designing programs for individual patients. Guidance on how to do this could be provided during dialysis. These recommendations coincide in many ways to the most recent PA guidelines for patients with chronic diseases [34]. A liberalized activity prescription such as this may result in greater participation and more sustained and robust changes in patients' lifestyles.

\section{Acknowledgment}

None.

\section{Disclosure Statement}

The authors have no conflicts of interest to declare.

\section{Funding Sources}

No funding sources were used in the preparation of this manuscript.

\section{Author Contributions}

K.R.W. directed all aspects of the literature review and manuscript preparation. H.-Y.F. took the lead in researching the scientific literature related to this topic, drafting the manuscript, including preparing the Table and Figure. B.T.B. and A.C.K. contributed significantly to the literature review, initial manuscript preparation, and subsequent revisions.

\section{References}

1 Johansen KL, Kaysen GA, Dalrymple LS, Grimes BA, Glidden DV, Anand S, et al. Association of physical activity with survival among ambulatory patients on dialysis: the Comprehensive Dialysis Study. Clin J Am Soc Nephrol. 2013 Feb;8(2):248-53.

2 Young HM, March DS, Graham-Brown MP, Jones AW, Curtis F, Grantham CS, et al. Effects of intradialytic cycling exercise on exercise capacity, quality of life, physical function and cardiovascular measures in adult haemodialysis patients: a systematic review and meta-analysis. Nephrol Dial Transplant. 2018 Aug;33(8):1436-45.

3 Chan D, Cheema BS. Progressive Resistance Training in End-Stage Renal Disease: systematic Review. Am J Nephrol. 2016;44(1):3245.
4 Kirkman DL, Mullins P, Junglee NA, Kumwenda M, Jibani MM, Macdonald JH. Anabolic exercise in haemodialysis patients: a randomised controlled pilot study. J Cachexia Sarcopenia Muscle. 2014 Sep;5(3):199-207.

5 Chan D, Green S, Fiatarone Singh M, Barnard $\mathrm{R}$, Cheema BS. Development, feasibility, and efficacy of a customized exercise device to deliver intradialytic resistance training in patients with end stage renal disease: non-randomized controlled crossover trial. Hemodial Int. 2016 Oct;20(4):650-60.

6 Dong J, Sundell MB, Pupim LB, Wu P, Shintani A, Ikizler TA. The effect of resistance exercise to augment long-term benefits of intradialytic oral nutritional supplementation in chronic hemodialysis patients. J Ren Nutr. 2011 Mar;21(2):149-59.
7 Kopple JD, Wang H, Casaburi R, Fournier M, Lewis MI, Taylor W, et al. Exercise in maintenance hemodialysis patients induces transcriptional changes in genes favoring anabolic muscle. J Am Soc Nephrol. 2007 Nov; 18(11) 2975-86.

8 Schardong J, Dipp T, Bozzeto CB, da Silva MG, Baldissera GL, Ribeiro RC, et al. Effects of Intradialytic Neuromuscular Electrical Stimulation on Strength and Muscle Architecture in Patients With Chronic Kidney Failure: Randomized Clinical Trial. Artif Organs. 2017 Nov;41(11):1049-58.

9 Pellizzaro CO, Thomé FS, Veronese FV. Effect of peripheral and respiratory muscle training on the functional capacity of hemodialysis patients. Ren Fail. 2013;35(2):18997. 
10 Bennett P, Corradini A, Ockerby C, Cossich T. Exercise during hemodialysis the intradialytic Zumba Gold. Nephrol News Issues. 2012 Aug;26(9):31-2.

11 Bennett PN, Parsons T, Ben-Moshe R, Weinberg M, Neal M, Gilbert K, et al. Laughter and humor therapy in dialysis. Semin Dial. 2014 Sep-Oct;27(5):488-93.

12 Clarkson MJ, Bennett PN, Fraser SF, Warmington SA. Exercise interventions for improving objective physical function in patients with end-stage kidney disease on dialysis: a systematic review and meta-analysis. Am J Physiol Renal Physiol. 2019 May;316(5):F856-72.

13 Koh KP, Fassett RG, Sharman JE, Coombes JS, Williams AD. Effect of intradialytic versus home-based aerobic exercise training on physical function and vascular parameters in hemodialysis patients: a randomized pilot study. Am J Kidney Dis. 2010 Jan;55(1):8899.

14 Manfredini F, Mallamaci F, D’Arrigo G, Baggetta R, Bolignano D, Torino C, et al. Exercise in Patients on Dialysis: A Multicenter, Randomized Clinical Trial. J Am Soc Nephrol. 2017 Apr;28(4):1259-68.

15 Han M, Ye X, Preciado P, Williams S, Campos I, Bonner $\mathrm{M}$, et al. Relationships between Neighborhood Walkability and Objectively Measured Physical Activity Levels in Hemodialysis Patients. Blood Purif. 2018;45(1-3): 236-44.

16 Molsted S, Harrison AP, Eidemak I, Andersen JL. The effects of high-load strength training with protein- or nonprotein-containing nutritional supplementation in patients undergoing dialysis. J Ren Nutr. 2013 Mar;23(2): $132-40$.

17 Nindl BC, Headley SA, Tuckow AP, Pandorf CE, Diamandi A, Khosravi MJ, et al. IGF-I system responses during 12 weeks of resistance training in end-stage renal disease patients. Growth Horm IGF Res. 2004 Jun; 14(3):245-50.

18 Headley S, Germain M, Mailloux P, Mulhern J, Ashworth B, Burris J, et al. Resistance training improves strength and functional mea- sures in patients with end-stage renal disease. Am J Kidney Dis. 2002 Aug;40(2):355-64.

19 Wilund KR, Jeong JH, Greenwood SA. Addressing myths about exercise in hemodialysis patients. Semin Dial. 2019 Jul;32(4):297302.

20 Tentori F, Elder SJ, Thumma J, Pisoni RL, Bommer J, Fissell RB, et al. Physical exercise among participants in the Dialysis Outcomes and Practice Patterns Study (DOPPS): correlates and associated outcomes. Nephrol Dial Transplant. 2010 Sep;25(9):3050-62.

21 Ma S, Lui J, Brooks D, Parsons TL. The availability of exercise rehabilitation programs in hemodialysis centres in Ontario. CANNT J. 2012 Oct-Dec;22(4):26-32.

22 McKenna CF, Salvador AF, Hendriks FK, Harris AP, van Loon LJ, Burd NA. Exercising to offset muscle mass loss in hemodialysis patients: the disconnect between intention and intervention. Semin Dial. 2019 Jul;32(4):379-85.

23 Greenwood SA, Lindup H, Taylor K, Koufaki P, Rush R, Macdougall IC, et al. Evaluation of a pragmatic exercise rehabilitation programme in chronic kidney disease. Nephrol Dial Transplant. 2012 Oct;27 Suppl 3:iii12634.

24 Delgado C, Johansen KL. Barriers to exercise participation among dialysis patients. Nephrol Dial Transplant. 2012 Mar;27(3): 1152-7.

25 Tawney KW, Tawney PJ, Hladik G, Hogan SL, Falk RJ, Weaver $\mathrm{C}$, et al. The life readiness program: a physical rehabilitation program for patients on hemodialysis. Am J Kidney Dis. 2000 Sep;36(3):581-91.

26 Sheng K, Zhang P, Chen L, Cheng J, Wu C, Chen J. Intradialytic exercise in hemodialysis patients: a systematic review and meta-analysis. Am J Nephrol. 2014;40(5):478-90.

27 Baggetta R, D’Arrigo G, Torino C, ElHafeez SA, Manfredini F, Mallamaci F, et al.; EXCITE Working group. Effect of a home based, low intensity, physical exercise program in older adults dialysis patients: a secondary analysis of the EXCITE trial. BMC Geriatr. 2018 Oct; 18(1):248.
28 Wilund KR, Viana JL, Perez LM. A Critical Review of Exercise Training in Hemodialysis Patients: Personalized Activity Prescriptions are Needed. Exerc Sport Sci Rev. 2019 Aug;1.

29 Chen JL, Godfrey S, Ng TT, Moorthi R, Liangos O, Ruthazer R, et al. Effect of intra-dialytic, low-intensity strength training on functional capacity in adult haemodialysis patients: a randomized pilot trial. Nephrol Dial Transplant. 2010 Jun;25(6):1936-43.

30 Johansen KL, Painter PL, Sakkas GK, Gordon P, Doyle J, Shubert T. Effects of resistance exercise training and nandrolone decanoate on body composition and muscle function among patients who receive hemodialysis: A randomized, controlled trial. J Am Soc Nephrol. 2006 Aug;17(8):2307-14.

31 Segura-Ortí E, Kouidi E, Lisón JF. Effect of resistance exercise during hemodialysis on physical function and quality of life: randomized controlled trial. Clin Nephrol. 2009 May; 71(5):527-37.

32 Cheema B, Abas H, Smith B, O'Sullivan A, Chan M, Patwardhan A, et al. Randomized controlled trial of intradialytic resistance training to target muscle wasting in ESRD: the Progressive Exercise for Anabolism in Kidney Disease (PEAK) study. Am J Kidney Dis. 2007 Oct;50(4):574-84.

33 Cheema B, Abas H, Smith B, O'Sullivan A, Chan M, Patwardhan A, et al. Progressive exercise for anabolism in kidney disease (PEAK): a randomized, controlled trial of resistance training during hemodialysis. J Am Soc Nephrol. 2007 May;18(5):1594-601.

34 Piercy KL, Troiano RP, Ballard RM, Carlson SA, Fulton JE, Galuska DA, et al.; The Physical Activity Guidelines for Americans. The Physical Activity Guidelines for Americans. JAMA. 2018 Nov;320(19):2020-8.

35 Jeong JH, Biruete A, Tomayko EJ, Wu PT, Fitschen P, Chung HR, et al. Results from the randomized controlled IHOPE trial suggest no effects of oral protein supplementation and exercise training on physical function in hemodialysis patients. Kidney Int. 2019 Sep;96(3):777-86. 